Research Article

\title{
Research on Output Signal Controlling of an Asymmetric Hydraulic Cylinder Based on a Flexible Connection
}

\author{
Tao Wang $\mathbb{D}$ and Jinchun Song $\mathbb{D}$ \\ School of Mechanical Engineering and Automation, Northeastern University, Shenyang 110819, Liaoning, China \\ Correspondence should be addressed to Tao Wang; molili94@126.com
}

Received 24 February 2021; Revised 21 March 2021; Accepted 23 March 2021; Published 5 April 2021

Academic Editor: Ricardo A. Ramirez-Mendoza

Copyright (c) 2021 Tao Wang and Jinchun Song. This is an open access article distributed under the Creative Commons Attribution License, which permits unrestricted use, distribution, and reproduction in any medium, provided the original work is properly cited.

In industrial production, the structures of hydraulic servo system-connecting device-load systems are often simplified as rigid connections for the ease of calculation. However, this simplification is problematic when applied to flexible connections in hydraulic systems, which generally have multivariable and strong couplings; these characteristics affect the control accuracy of the hydraulic servo system and lead to serious distortion of the output waveform, which cannot be ignored. These problems cause greater lag and attenuation of the actual signal than those of the expected signal, leading to lower credibility. Therefore, it is important to study the waveform distortion caused by flexible connections. In this paper, according to the characteristics of a flexible connection, a corresponding mathematical model is established, and an adaptive controller, whose structure is simple and calculation cost is low, is used to adjust the amplitude and phase of the response signal and improve the accuracy of the system response. Treating the change in the response signal as the error value, the algorithm weights are adjusted until the error value is stable. Then, a more accurate output signal is obtained. Finally, the validity and practicability of the adaptive controller are verified by simulation experiments.

\section{Introduction}

Due to the application of servo valves, hydraulic servo systems are gradually being widely applied in industry. Generally, the displacement and control quantity are the controlled quantities of hydraulic servo systems. The control system is required to have highly dynamic characteristics and to reproduce the input signal accurately, which makes it a crucial component of industrial equipment and other applications $[1,2]$. To facilitate the design and application of systems in related fields, the structure of a hydraulic servo system-connecting device-load system is often adopted, and it is often regarded as an entirely rigid system. That is, under this assumption, the middle connecting structure of the mechanical system does not deform, and its corresponding elastic coefficient is considered to be infinite. Although this simplified form offers convenience, the elasticity cannot be ignored in specific situations. Elasticity not only causes deterioration of the control performance and unexpected oscillation in the output but also increases the adjustment time and even causes loss of system stability. This leads to failure in obtaining accurate system position and speed data and affects the control condition $[3,4]$.

Nonlinear factors such as friction, clearance, and dead zones often reduce the accuracy and even cause instability. For electrohydraulic servo systems, various mechanical, hydraulic, electronic, and other nonlinear phenomena are integrated into one phenomenon, the flow-pressure characteristic. In hydraulic systems, the parameters related to the flow rate, pressure, and viscosity of oil vary with time $[5,6]$. Therefore, when the electrohydraulic servo system is excited by a sine signal, the higher harmonics appear in the acceleration output, which seriously distorts the signal [7]. In addition, due to servo valve manufacturing, adjustment, and assembly error, zero offset is often inevitable. Although usually adjusted to a minimum, zero offset typically increases due to looseness or distortion of some centering components caused by vibration. For high-precision and 
high-response systems, zero offset reduces the accuracy and response speed of the system. The influence of friction nonlinearity on the hydraulic system is also very complex, and Coulomb friction causes dead zone characteristics. At low speed, viscous friction produces additional nonlinearity such as hysteresis, while at high speed, the influence of viscous friction can be ignored.

In practical applications, the changes in load and flexible connections limit the improvement potential of hydraulic servo system performance. In the traditional design, the control parameters of the hydraulic servo system do not change with the load inertia. When the load inertia changes, the performance deteriorates. Therefore, realizing a fast response or high dynamic stiffness of the system is a common problem. Many theoretical studies have been performed on the optimization of the flexible connection load control. The research in this field involves a variety of control methods. Commonly used traditional methods include fuzzy control and PID robust control. In addition, there are many intelligent algorithms such as the network control, adaptive control, repetitive control, and genetic algorithm that provide a theoretical basis for solving problems [8-11]. Lee et al. proposed a controller algorithm using state observer to monitor the change in disturbance torque to realize signal tracking at the load side [12]. Umeno et al. generated a robust speed control system of a servo motor based on a parametric two-degrees-of-freedom controller, which improved the input response performance of the system [13]. Nayyerloo et al. studied the nonlinear structure under vibration and proposed an improved LMSbased SHM algorithm to estimate the output velocity signal more accurately and its influence on vibration suppression [14]. For a class of high-order nonlinear systems with mismatched uncertain parameters and external loads, Guan and Zhu proposed a multisliding mode-based robust adaptive control strategy to reduce the tracking error caused by system uncertainty [15]. Rozaimi et al. used sliding mode control (SMC) to evaluate the position tracking performance of an electrohydraulic servo (EHS) system, an approach that was established in the process of considering the nonlinear friction model [16].

Although the aforementioned methods can improve the performance of the output signal, they seldom consider the control situation of flexible connections based on asymmetric actuators. In this paper, the asymmetric hydraulic cylinder servo mechanism is used as an experimental platform to construct a two-mass flexible connection to improve the accuracy of the servo mechanism response signal and a servo control strategy based on adaptive control is studied. The paper is primarily focused on the two-mass flexible connection state of an asymmetric hydraulic cylinder, for which the corresponding mathematical model is established. The mathematical factors of the coupling phenomenon can be understood from the model. The adaptive algorithm formula is used to construct an adaptive output control to ensure the accuracy of the output signal. From the derived adaptive formula, it is seen that the value of the step size parameter is important to determine for accurate control. Through simulations and experiments before and after the input of the sinusoidal signal, the fuzzy PID algorithm is compared with the adaptive algorithm. It is proven that the adaptive output control has good reproducibility for the signal and effectively reproduces the amplitude and phase characteristics of the input signal without identifying the system model. The adaptive output control is not specific to a certain kind of nonlinearity. In the application, the adaptive algorithm needs only to adjust the step size. Therefore, it has advantages of low computation costs, a simple structure, and wide application range.

\section{Mathematical Modeling of Asymmetric Cylinder with Two-Mass Flexible Connection}

Asymmetric hydraulic cylinders have advantages such as small space, a simple structure, convenient operation, and low cost. Therefore, asymmetric actuators have been effectively applied to some specific hydraulic control systems such as multipoint linkage compound hydraulic loading control systems, fatigue strength simulation tests of mechanical vibration parts, simulation operations of driving control systems, and simulation operation tests of hydraulic vibration systems based on servo mechanical shaking tables.

A sketch of the asymmetric hydraulic cylinder system including various parameters is shown in Figure 1. Ignoring the influence of friction on the load, the system is regarded as a two-mass system model for analysis under the effect of a flexible connection. It is controlled by an ideal zero-opening four-sided slide valve, and the hydraulic cylinder leakage and compressibility of the hydraulic oil are not taken into account. By default, the hydraulic cylinder load remains constant, the supply pressure remains unchanged, and the return oil pressure is zero. The mathematical modeling takes the research object of the hydraulic system as the main condition, while the secondary condition is not considered.

Considering the convenience and main factors of actual hydraulic modeling, it is necessary to make the following assumptions: there is no pressure loss in the pipeline; the pressure loss at the local and inflection points is not considered; the hydraulic oil temperature is in an ideal state; the hydraulic oil density is constant or the changes are small enough to be ignored; and the internal or external leakage is in a laminar flow state.

According to the actual model of an asymmetric hydraulic cylinder, the selected ratio is $a=\left(A_{2} / A_{1}\right)$; therefore, the flow relationship between the left and right chambers is

$$
q_{2}=a q_{1}
$$

Based on hydraulic industry regulations, the right movement of the valve core is in a positive direction, and the movement direction of the valve core is divided into two kinds of motion situations. When the spool moves in the right direction, that is, when the spool displacement $x_{\mathrm{v}}>0$, the linearized flow continuity equation is

$$
q_{L}=K_{q} x_{v}-K_{c} p_{L}
$$

When the slide valve moves in the left direction, that is, when the displacement $x_{\mathrm{v}}<0$, the piston is in the situation of 


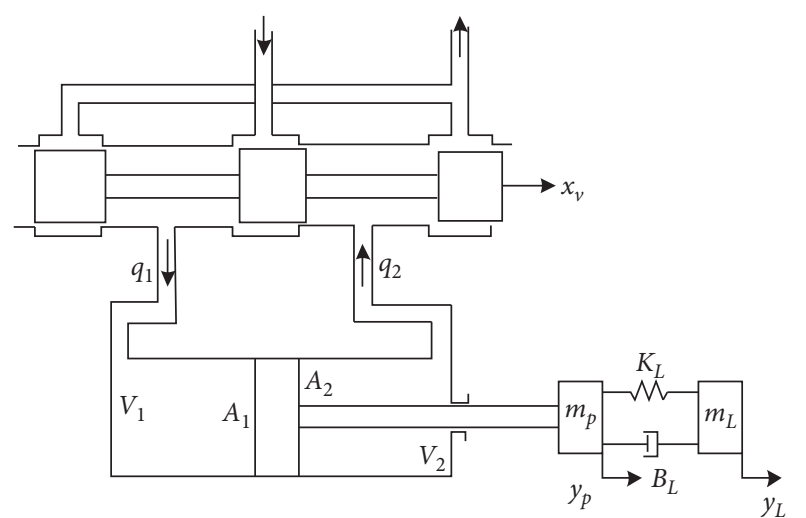

Figure 1: The model of the asymmetric cylinder system with flexible connection.

a left retraction. The linear flow continuity equation of the retraction motion is

$$
q_{L}^{\prime}=K_{q}^{\prime} x_{v}-K_{c}^{\prime} p_{L}^{\prime}
$$

The flow equation in the two cavities of the hydraulic cylinder under ideal conditions is as follows. When the slide valve moves in the right direction, the friction and pressure loss of the hydraulic servo system are not considered. The quality factor of liquid flow is ignored.

$$
\left\{\begin{array}{l}
q_{1}=C_{i p}\left(p_{1}-p_{2}\right)+C_{e p} p_{1}+\frac{V_{1}}{\beta_{e}} \frac{\mathrm{d} p_{1}}{\mathrm{~d} t}+A_{1} \frac{\mathrm{d} y}{\mathrm{~d} t}, \\
q_{2}=C_{i p}\left(p_{1}-p_{2}\right)-C_{e p} p_{2}-\frac{V_{2}}{\beta_{e}} \frac{\mathrm{d} p_{2}}{\mathrm{~d} t}+A_{2} \frac{\mathrm{d} y}{\mathrm{~d} t} .
\end{array}\right.
$$

Based on the possible leakage of hydraulic parts and the compressibility of hydraulic oil, the equations of flow and load are established as follows:

$$
q_{L}=C_{i p}\left(p_{1}-p_{2}\right)+C_{e p} p_{1}+\frac{1+a^{2}}{1+a^{3}} \frac{V_{e}}{\beta_{e}} \frac{\mathrm{d} p_{L}}{\mathrm{~d} t}+\left(\frac{A_{1}+A_{2}}{2}\right) \frac{\mathrm{d} y}{\mathrm{~d} t} .
$$

For the whole hydraulic system, the internal leakage coefficient is very small; therefore, its influence can be almost ignored, and the simplified equation is obtained as follows:

$$
q_{L}=C_{t c} p_{L}+\frac{1+a^{2}}{1+a^{3}} \frac{V_{e}}{\beta_{e}} \frac{\mathrm{d} p_{L}}{\mathrm{~d} t}+\left(\frac{A_{1}+A_{2}}{2}\right) \frac{\mathrm{d} y}{\mathrm{~d} t} .
$$

Because the equivalent volume $V_{e}$ is a function of displacement, it is necessary to simplify the constant in order to obtain a linear expression of the flow rate.

$$
\left\{\begin{array}{l}
q_{1}^{\prime}=C_{i p}^{\prime}\left(p_{1}^{\prime}-p_{2}^{\prime}\right)-C_{e p}^{\prime} p_{1}^{\prime}-\frac{V_{1}}{\beta_{e}} \frac{\mathrm{d} p_{1}^{\prime}}{\mathrm{d} t}+A_{1} \frac{\mathrm{d} y}{\mathrm{~d} t}, \\
q_{2}^{\prime}=C_{i p}^{\prime}\left(p_{1}^{\prime}-p_{2}^{\prime}\right)+C_{e p}^{\prime} p_{2}^{\prime}+\frac{V_{2}}{\beta_{e}} \frac{\mathrm{d} p_{2}^{\prime}}{\mathrm{d} t}+A_{2} \frac{\mathrm{d} y}{\mathrm{~d} t} .
\end{array}\right.
$$

Similarly, considering the possible leakage of hydraulic parts and the compressibility of hydraulic oil, the equation of the load flow and load is established as follows:

$$
q_{L}^{\prime}=C_{i p}^{\prime}\left(p_{1}^{\prime}-p_{2}^{\prime}\right)+C_{e p}^{\prime} p_{2}^{\prime}+\frac{1+a^{2}}{1+a^{3}} \frac{V_{e}^{\prime}}{\beta_{\mathrm{e}}} \frac{\mathrm{d} p_{2}^{\prime}}{\mathrm{d} t}+\left(\frac{A_{1}+A_{2}}{2}\right) \frac{\mathrm{d} y}{\mathrm{~d} t} .
$$

The equation relating load flow, load pressure, and hydraulic oil source is obtained as follows:

$$
q_{L}^{\prime}=C_{t c}^{\prime} p_{L}^{\prime}+\frac{1+a^{2}}{1+a^{3}} \frac{V_{e}^{\prime}}{\beta_{e}} \frac{\mathrm{d} p_{L}^{\prime}}{\mathrm{d} t}+\left(\frac{A_{1}+A_{2}}{2}\right) \frac{\mathrm{d} y}{\mathrm{~d} t} .
$$

Considering the effect of the viscous damping force, the force balance equation based on two-degrees-of-freedom is as follows:

$$
\left\{\begin{array}{l}
A_{1} p_{1}-A_{2} p_{2}=m_{p} \frac{\mathrm{d}^{2} y_{p}}{\mathrm{~d} t^{2}}+B_{L} \frac{\mathrm{d} y_{p}}{\mathrm{~d} t}+K_{L} y_{P}-B_{L} \frac{\mathrm{d} y_{L}}{\mathrm{~d} t}-K_{L} y_{L} \text { right, } \\
A_{2} p_{2}-A_{1} p_{1}=m_{p} \frac{\mathrm{d}^{2} y_{p}}{\mathrm{~d} t^{2}}+B_{L} \frac{\mathrm{d} y_{p}}{\mathrm{~d} t}+K_{L} y_{P}-B_{L} \frac{\mathrm{d} y_{L}}{\mathrm{~d} t}-K_{L} y_{L} \text { left, }
\end{array}\right.
$$$$
m_{L} \frac{\mathrm{d}^{2} y_{L}}{\mathrm{~d} t^{2}}=B_{L} \frac{\mathrm{d} y_{p}}{\mathrm{~d} t}+K_{L} y_{P}-B_{L} \frac{\mathrm{d} y_{L}}{\mathrm{~d} t}-K_{L} y_{L} .
$$

According to the previous formula, the Laplace transform is carried out and sorted out.

$$
\left\{\begin{array}{l}
A_{1} P_{1}-A_{2} P_{2}=m_{p} s^{2} Y_{p}+B_{L} s Y_{p}+K_{L} Y_{P}-B_{L} s Y_{L}-K_{L} Y_{L} \text { right, } \\
A_{2} P_{2}-A_{1} P_{1}=m_{p} s^{2} Y_{p}+B_{L} s Y_{p}+K_{L} Y_{P}-B_{L} s Y_{L}-K_{L} Y_{L} \text { left, }
\end{array}\right.
$$

$$
m_{L} s^{2} Y_{L}=B_{L} s Y_{p}+K_{L} Y_{P}-B_{L} s Y_{L}-K_{L} Y_{L} .
$$

When the pressure oil inflows to the rodless side of the hydraulic cylinder, the piston is driven to extend out, and expressions corresponding to equations (2), (6), (11), and (12) are obtained as follows:

$$
\begin{aligned}
\frac{Y_{p}}{X_{V}}= & \frac{K_{q}}{A_{1}} \frac{m_{L} s^{2}+B_{L} s+K_{L}}{D(s)}, \\
D(s)= & \frac{1+a^{2}}{1+a^{3}} \frac{m_{L} m_{p}}{K_{h}} s^{5}+\left[\frac{1+a^{2}}{1+a^{3}} \frac{B_{L}\left(m_{L}+m_{p}\right)}{K_{h}}+\frac{K_{c e} m_{L} m_{p}}{A_{1}^{2}}\right] s^{4} \\
& +\left[\frac{a+1}{2} m_{L}+\frac{K_{c e} B_{L}\left(m_{L}+m_{p}\right)}{A_{1}^{2}}+\frac{1+a^{2}}{1+a^{3}} \frac{K_{L}\left(m_{L}+m_{p}\right)}{K_{h}}\right] s^{3} \\
& +\left[\frac{a+1}{2} B_{L}+\frac{K_{c e} K_{L}\left(m_{L}+m_{p}\right)}{A_{1}^{2}}\right] s^{2}+\frac{a+1}{2} K_{L} s .
\end{aligned}
$$

Similarly, when the spool valve core moves to the left and the piston of the hydraulic cylinder retracts, by comparing equations (3), (9), (11), and (12), the following expressions are obtained: 


$$
\begin{aligned}
\frac{Y_{p}}{X_{V}}= & \frac{K_{q}^{\prime}}{A_{1}} \frac{a\left(m_{L} s^{2}+B_{L} s+K_{L}\right)}{D(s)}, \\
D(s)= & \frac{1+a^{2}}{1+a^{3}} \frac{m_{L} m_{p}}{K_{h}^{\prime}} s^{5}+\left[\frac{1+a^{2}}{1+a^{3}} \frac{B_{L}\left(m_{L}+m_{p}\right)}{K_{h}^{\prime}}+\frac{K_{c e}^{\prime} m_{L} m_{p}}{A_{1}^{2}}\right] s^{4} \\
& +\left[\frac{a+1}{2} m_{L}+\frac{K_{c e}^{\prime} B_{L}\left(m_{L}+m_{p}\right)}{A_{1}^{2}}+\frac{1+a^{2}}{1+a^{3}} \frac{K_{L}\left(m_{L}+m_{p}\right)}{K_{h}^{\prime}}\right] s^{3} \\
& +\left[\frac{a+1}{2} B_{L}+\frac{K_{c e}^{\prime} K_{L}\left(m_{L}+m_{p}\right)}{A_{1}^{2}}\right] s^{2}+\frac{a+1}{2} K_{L} s .
\end{aligned}
$$

From this analysis, it is seen that the coupling effect of the hydraulic system constitutes internal resonance. Compared with the mathematical formula of an inertial system, the mathematical formula of a hydraulic system with a flexible connection adds two links, namely, an oscillation link and a second-order differential link. Due to the uncertainty and nonlinearity of the coupling characteristics, it causes oscillations in the system bandwidth.

\section{Research on Control Strategy}

3.1. Background of the Problem. A sinusoidal signal is commonly used as a given signal in hydraulic control. However, due to the influence of nonlinear factors of a hydraulic system and the limitation of hydraulic valve characteristics, the closed-loop frequency characteristics of the hydraulic system are inferior, which make it is impossible to obtain an accurate output signal. The influence of the servo valve dead zone and flow nonlinearity on the system leads to large waveform distortion and phase lag. Both the positive overlap of the slide valve and the Coulomb friction cause a dead zone characteristic, which has a great influence on the static error of the system but generally does not damage its stability. The dead zone characteristic delays the response of the system, which is the main reason for the distortion of the system response waveform. Therefore, the peak value of the output sinusoidal response signal deviates. Even when a feedforward control is used, the sinusoidal signal cannot be accurately obtained.

In a servo hydraulic system, the sinusoidal response signal often leads to phase lag and amplitude attenuation, especially phase lag; neither high tracking accuracy of the sinusoidal response signal at each frequency point nor reaching the specified signal amplitude or phase is guaranteed [17]. Therefore, to realize the method of embedded controller, the amplitude and phase of the fundamental frequency response should be adjusted necessarily.

3.2. Fuzzy PID Control. Generally, the fuzzy PID controller is used as a control method. It takes the variable error $e$ and error change $e c$ as the inputs. The error signal $e$ and its change rate $\mathrm{d} e / \mathrm{d} t$ are obtained by using fuzzy rules in the feedback system to calculate the control quantity $[18,19]$.
The structure of a typical adaptive fuzzy PID controller is shown in Figure 2.

The setting process of the algorithm is summarized by three standard rules as follows:

(1) When the error value $e$ is too large, it is necessary to accelerate the response speed of the system. To avoid overshooting the system, a smaller $K_{i}$ value is chosen and the value of $K_{p}$ is increased as much as possible.

(2) When the error value $e$ is moderate, to prevent excessive overshoot, a smaller $K_{p}$ value is usually selected. In addition, it is necessary to select a value in line with $K_{i}$ and $K_{d}$; otherwise, the running speed of the system is affected.

(3) When the error value $e$ is small, the selected $K_{p}$ and $K_{i}$ values should be increased to a certain extent to meet stability requirements. It is also necessary to select an appropriate value of $K_{d}$ according to the error change rate; when the error change rate is small, the value of $K_{d}$ is generally moderate to meet the requirements of the system. When the error change rate is large, $K_{d}$ needs to be smaller.

In the actual operation process, the basic process of fuzzy PID control parameter tuning is shown in Figure 3. The fuzzy PID algorithm is based on the error and error rate of change, which are used to calculate the values of the adjustment control quantities $\Delta K_{p}, \Delta K_{i}$, and $\Delta K_{d}$. The type of the controller is the input of two parameters and the output structure of three parameters. The structure of the constructed fuzzy controller is shown in Figure 4. In the process of fuzzification and defuzzification, a membership function is used as a tool to establish the relationship between the control parameters and control rules. Through the fuzzy universe of parameter variables in the algorithm, the definitions of error $e$, error rate $e c, \Delta K_{p}, \Delta K_{i}$, and $\Delta K_{d}$ are determined and expressed as a fuzzy set $\{\mathrm{NB}, \mathrm{NM}, \mathrm{NS}, \mathrm{ZO}$, PS, PM, PB\}, as shown in Table 1. Finally, the fuzzy controller is set up after the rule table is established [20, 21].

Although the fuzzy PID algorithm has many advantages, it has high requirements for control rules, a lack of systematic control design, and limited application scope. The simple fuzzy processing of information often leads to a reduction in control accuracy and the deterioration of dynamic quality, and the control purpose cannot be defined. Solving the problem of multivariable coupling and higherorder functions is not ideal. Therefore, it is necessary to introduce better methods to solve complex problems in hydraulic servo systems [22].

3.3. Adaptive Filter Theory. To achieve higher output accuracy and ensure an online embedded controller to implement the control strategy, a control strategy based on linear adaptive filter theory is a more feasible scheme.

A detailed block diagram of a transverse filter that realizes the whole filtering process under the control of the adaptive control algorithm is shown in Figure 5. The tap input is the sequence of input vectors $\mathbf{u}(n)$ representing $M \times 1$, and the tap weight is the sequence of matrix $M \times 1$ tap 


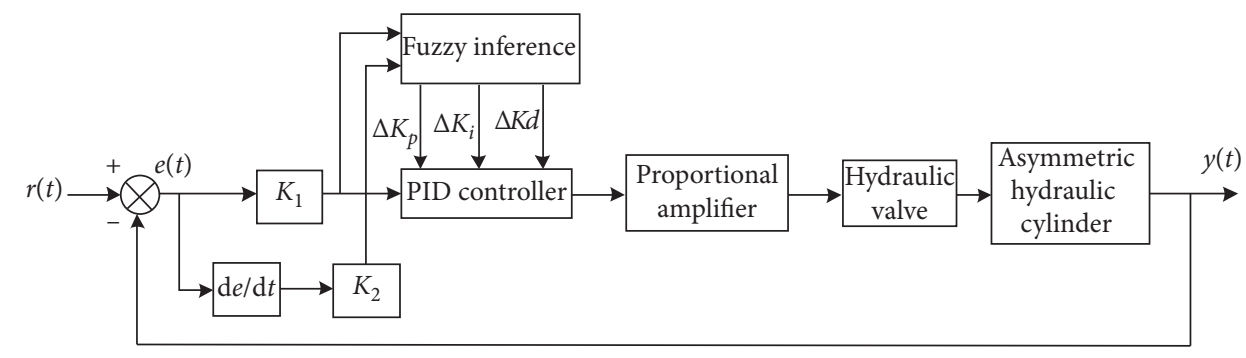

FIgURe 2: Schematic diagram of fuzzy PID control.

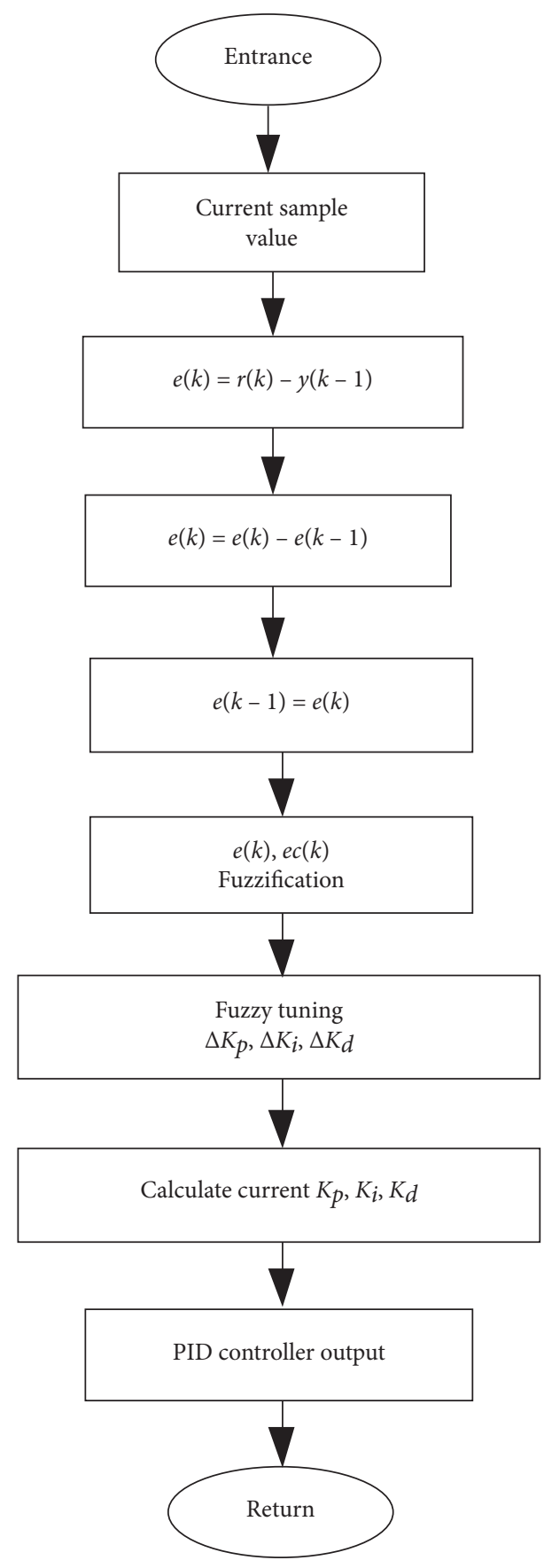

Figure 3: Flow chart of the fuzzy PID control algorithm.

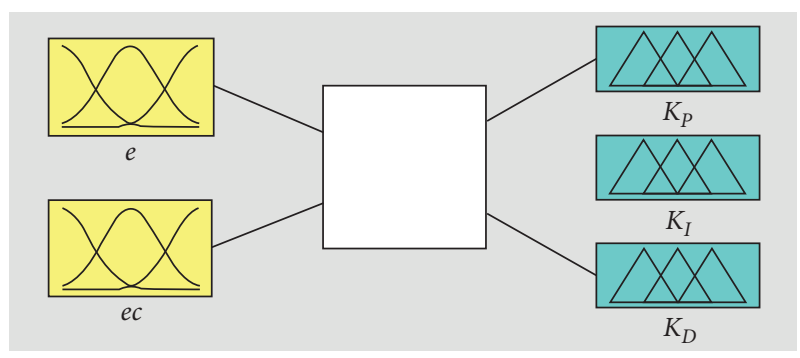

FIgURE 4: Structure of fuzzy controller.

weight vectors $\widehat{w}_{0}(n)$. To obtain an estimated expected value that is close to the value of the Wiener solution, the number of iterations tends to infinity. Under the given input, the output is the estimated value of the expected response $\widehat{d}(n)$, that is, the estimated value of the expected response $d(n)$. In fact, the difference between the output of the filter and the expected response is defined as the estimation error, and the estimation error $e(n)$ and the tap input vector $u(n)$ are inputs of the adaptive algorithm, thus forming closed-loop feedback $[23,24]$.

Assuming that the step size parameter is selected to take the value of $\mu$ appropriately and the gradient vector $\nabla \mathbf{J}(n)$ of each iteration $n$ is accurately measured, then the tap vector will converge to the Wiener solution [25, 26]. The estimation formula of the gradient vector $\nabla \mathbf{J}(n)$ is as follows:

$$
\nabla \mathbf{J}(n)=-2 \mathbf{p}+2 \mathbf{R} \mathbf{w}(n) .
$$

As the transient estimation of the expected response and tap input vector, the expression is as follows:

$$
\begin{aligned}
\widehat{\mathrm{R}}(n) & =\mathbf{u}(n) \mathbf{u}^{H}(n), \\
\widehat{\mathrm{P}}(n) & =\mathbf{u}(n) d^{*}(n) .
\end{aligned}
$$

Superscript H means Hermitian transpose and * means complex conjugate. ^represents the tap weight vector. The transient estimation of gradient vector is generally biased, and the expression is as follows:

$$
\hat{\nabla} \mathbf{J}(n)=-2 \mathbf{u}(n) d^{*}(n)+2 \mathbf{u}(n) \mathbf{u}^{H}(n) \widehat{w}(n) .
$$

Based on this, a new formula to update the tap vector is obtained. The recursive formula is as follows:

$$
\widehat{\mathrm{w}}(n+1)=\widehat{w}(n)+\mu \mathbf{u}(n)\left[d^{*}(n)-\mathbf{u}^{H}(n) \widehat{w}(n)\right] .
$$


TABLE 1: Representation meaning of parameters in fuzzy sets.

\begin{tabular}{|c|c|c|c|c|c|c|c|}
\hline Symbol & NB & NM & NS & $\mathrm{ZO}$ & PS & PM & $\mathrm{PB}$ \\
\hline Meaning & Negative big & Negative middle & Negative small & Zero & Positive small & Positive middle & Positive big \\
\hline
\end{tabular}

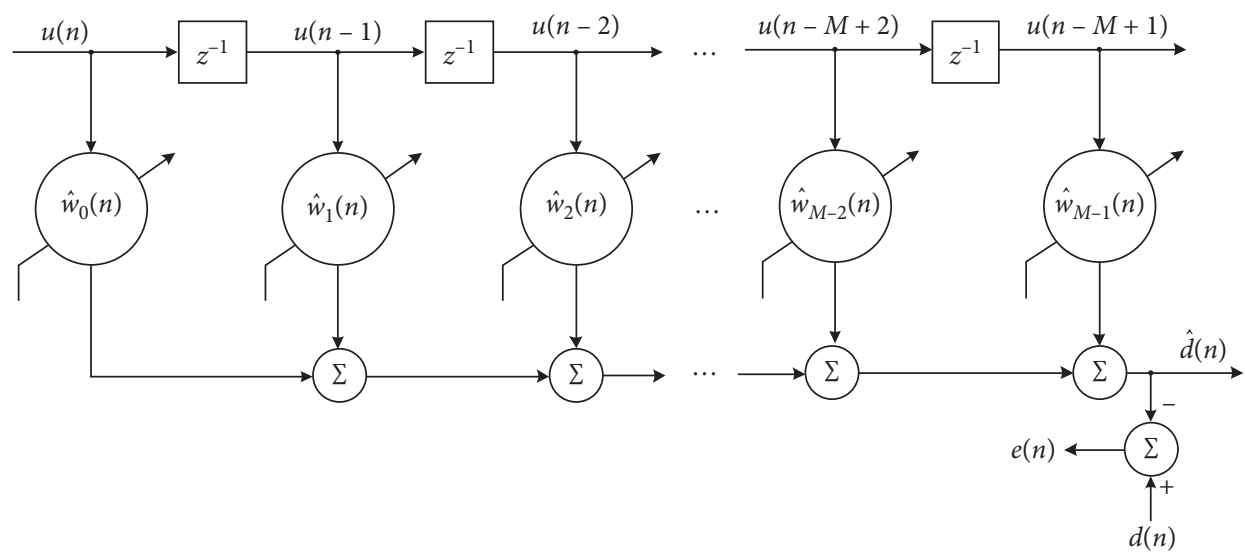

FIGURE 5: Block diagram of transverse filter structure.

Set the tap input vector of transverse filter as

$$
\mathbf{u}(n)=[u(n), u(n-\tau)]^{T}=[A \sin (\omega n), A \cos (\omega n)]^{T},
$$

where $\tau$ is the sampling time of $\cos (\omega n)$ lagging $\sin (\omega n)$. The output of transverse filter is as follows:

$$
\mathbf{u}_{c}(n)=\widehat{w}^{H}(n) \mathbf{u}(n),
$$

where $\widehat{w}=\left[w_{1} w_{2}\right]^{T}$. Equation (19) is introduced into equation (20) to obtain the following:

$$
\begin{aligned}
u_{c}(n) & =w_{1} A \sin (\omega n)+w_{2} A \cos (\omega n) \\
& =A \sqrt{w_{1}^{2}+w_{2}^{2}}\left[\frac{w_{1}}{\sqrt{w_{1}^{2}+w_{2}^{2}}} \sin (\omega n)+\frac{w_{2}}{\sqrt{w_{1}^{2}+w_{2}^{2}}} \cos (\omega n)\right] .
\end{aligned}
$$

Make

$$
\cos (\varphi)=\left(w_{1} / \sqrt{w_{1}^{2}+w_{2}^{2}}\right), \sin (\varphi)
$$

$=\left(w_{2} / \sqrt{w_{1}^{2}+w_{2}^{2}}\right)$, and $A_{c}=A \sqrt{w_{1}^{2}+w_{2}^{2}}$, so it is obtained that

$$
u_{c}(n)=A_{c} \sin (\omega n+\varphi)
$$

According to the formula described above, as long as the input signal obtains the corresponding phase advance $\varphi$, the phase lag of system can be eliminated. The output amplitude and phase of the adaptive controller are changed relative to the original sinusoidal signal by altering the values of $w_{1}$ and $w_{2}$, and the output is compensated.

The block diagram of amplitude and phase controller for adjusting weights using the standard adaptive algorithm is shown in Figure 6, where the difference between the actual responses and expected responses of sine is used as the signal of the algorithm. When the mean square error value reaches the minimum value, in terms of mean square error, the amplitude and phase compensation of the adaptive controller also reach the best value. That is, the input signal can be reproduced accurately. The iterative formulas of adaptive controller are summarized as follows:

$$
\begin{aligned}
u_{c}(n) & =\widehat{w}^{H}(n) \mathbf{u}(n), \\
e(n) & =u(n)-a(n), \\
\widehat{w}(n+1) & =\widehat{w}(n)+\mu \mathbf{u}(n) e(n) .
\end{aligned}
$$

For the stability of the adaptive algorithm, the eigenvalues of the correlation matrix of the correlation matrix are closely related to the adaptive algorithm. For the input vector of equation (19), the corresponding characteristic equation is written as

$$
R=\left|\begin{array}{cc}
E\left[u^{2}(n)\right] & E[u(n) u(n-\tau)] \\
E[u(n-\tau) u(n)] & E\left[u^{2}(n-\tau)\right]
\end{array}\right|
$$

For the value of $E\left[u^{2}(t)\right]$ and $E[u(t-\tau) u(t)], u(t)$ is a periodic signal and $E[u(t)]=\lim _{T \rightarrow \infty}(1 / T) \int_{-T \Omega}^{T \Omega} u(t) \mathrm{d} t$, so there is

$$
\begin{aligned}
E[u(t) u(t-\tau)] & =E\left[A^{2} \sin (\omega t) \cos (\omega t)\right] \\
& =A^{2} \lim _{T \rightarrow \infty} \frac{1}{T} \int_{-(1 / T)}^{(1 / T)} \sin (\omega t) \cos (\omega t) \mathrm{d} t=0 .
\end{aligned}
$$

From this, a conclusion is obtained:

$$
E[u(n-\tau) u(n)]=E[u(n) u(n-\tau)] .
$$




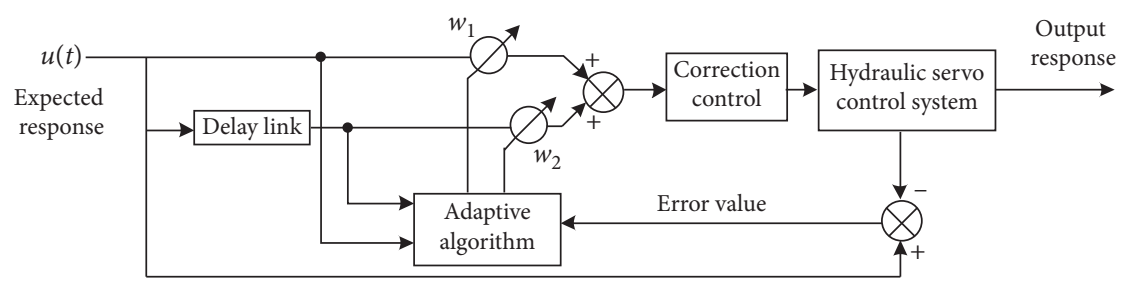

FIGURE 6: Controller with weight adjustment based on the adaptive algorithm.

For the same principle, it is obtained that

$$
\begin{aligned}
E\left[u^{2}(t)\right] & =E\left[A^{2} \sin ^{2}(\omega t)\right] \\
& =A^{2} \lim _{T \rightarrow \infty} \frac{1}{T} \int_{-(1 / T)}^{(1 / T)} \sin ^{2}(\omega t)=\frac{A^{2}}{2} .
\end{aligned}
$$

It is seen from equation (27) that

$$
E\left[u^{2}(n)\right]=E\left[u^{2}(n-\tau)\right]=\frac{A^{2}}{2}
$$

The correlation matrix can be obtained by substituting equations (26) and (28) into equation (24)

$$
\mathbf{R}=\left|\begin{array}{cc}
\frac{A^{2}}{2} & 0 \\
0 & \frac{A^{2}}{2}
\end{array}\right| .
$$

The eigenvalue of the matrix is obtained as follows:

$$
\lambda_{1,2}=\frac{A^{2}}{2} \text {. }
$$

Combining the output of $y(n)=\widehat{\mathbf{w}^{H}}(n) \mathbf{u}(n)$ with estimation error $e(n)=d(n)-y(n)$ and the definition of the weight error vector, the estimation error is as follows:

$$
\begin{aligned}
e(n) & =d(n)-\widehat{\mathbf{w}^{H}}(n) \mathbf{u}(n) \\
& =d(n)-\mathbf{w}_{0}^{H}(n)+\boldsymbol{\varepsilon}^{H}(n) \mathbf{u}(n) \\
& =e_{0}(n)+\boldsymbol{\varepsilon}^{H}(n) \mathbf{u}(n) \\
& \approx e_{0}(n)+\boldsymbol{\varepsilon}_{0}^{H}(n) \mathbf{u}(n) .
\end{aligned}
$$

The sign $\varepsilon_{0}(n)$ in the formula represents the weight error vector, so its mean square error is as follows:

$$
\begin{aligned}
J(n)= & E\left[|e(n)|^{2}\right] \\
\approx & E\left\{\left[e_{0}(n)+\boldsymbol{\varepsilon}_{0}^{H}(n) \mathbf{u}(n)\right]\left[e_{0}^{*}(n)+\mathbf{u}^{H}(n) \boldsymbol{\varepsilon}_{0}(n)\right]\right\} \\
= & J_{\min }+2 \operatorname{Re}\left\{E\left[e_{0}^{*}(n) \boldsymbol{\varepsilon}_{0}^{H}(n) \mathbf{u}(n)\right]\right\} \\
& +E\left[\boldsymbol{\varepsilon}_{0}^{H}(n) \mathbf{u}(n) \mathbf{u}^{H}(n) \boldsymbol{\varepsilon}_{0}(n)\right] .
\end{aligned}
$$

According to the small step hypothesis, it is known that

$$
E\left[e_{0}^{*}(n) \varepsilon_{0}^{H}(n) \mathbf{u}(n)\right]=0 .
$$

It is deduced from the same principle that

$$
E\left[\boldsymbol{\varepsilon}_{0}^{H}(n) \mathbf{u}(n) \mathbf{u}^{H}(n) \boldsymbol{\varepsilon}_{0}(n)\right]=\sum_{k 1}^{M} \lambda_{k} E\left[\left|v_{k}(n)\right|^{2}\right] .
$$

$v_{k}(n)$ is expressed as the $k$ th natural mode of the filter [27]. By substituting equations (33) and (34) into equation (32), the following is obtained:

$$
\begin{aligned}
J(n)= & J_{\min }+\mu J_{\min } \sum_{k=1}^{M} \frac{\lambda_{k}}{2-\mu \lambda_{k}} \\
& +\sum_{k=1}^{M} \lambda_{k}\left(\left|v_{k}(0)\right|^{2}-\frac{\mu J_{\min }}{2-\mu \lambda_{k}}\right)\left(1-\mu \lambda_{k}\right)^{2 n} .
\end{aligned}
$$

When the value $\mu$ is small, equation (35) is simplified as

$$
J(n) \approx J_{\min }+\mu J_{\min } \sum_{k=1}^{M} \lambda_{k}+\sum_{k=1}^{M} \lambda_{k}\left(\left|v_{k}(0)\right|^{2}-\frac{\mu J_{\min }}{2}\right)\left(1-\mu \lambda_{k}\right)^{2 n} \text {. }
$$

The mean square value of estimation error consists of two parts: the independency depends on the steady-state component of iteration number $n$ and the transient component. The cost function $J(n)$ at $n$ time is controlled by an exponential factor $\left(1-\mu \lambda_{k}\right)^{2 n}$. When the step size is small, the exponential factor $\left(1-\mu \lambda_{k}\right)^{2 n}$ will decay to zero as the iteration increases $[28,29]$. In this case, the learning curve decays to a constant value, and there is no oscillation. The specific expression is as follows:

$$
J(\infty) \approx J_{\min }+\frac{\mu J_{\min }}{2} \sum_{k=1}^{M} \lambda_{K}
$$

Therefore, it can be seen from equation (37) that $\mu$ must satisfy the following inequality to make the cost function converge:

$$
0<\mu<\frac{2}{\lambda_{\max }}
$$

where $\lambda_{\max }$ is the largest eigenvalue of the tap vector correlation matrix R. According to formula (38), the necessary conditions for stability are as follows:

$$
\mu<\frac{4}{A^{2}}
$$

which is a necessary condition for the adaptive algorithm to achieve convergence, so in order to ensure the safety, the 


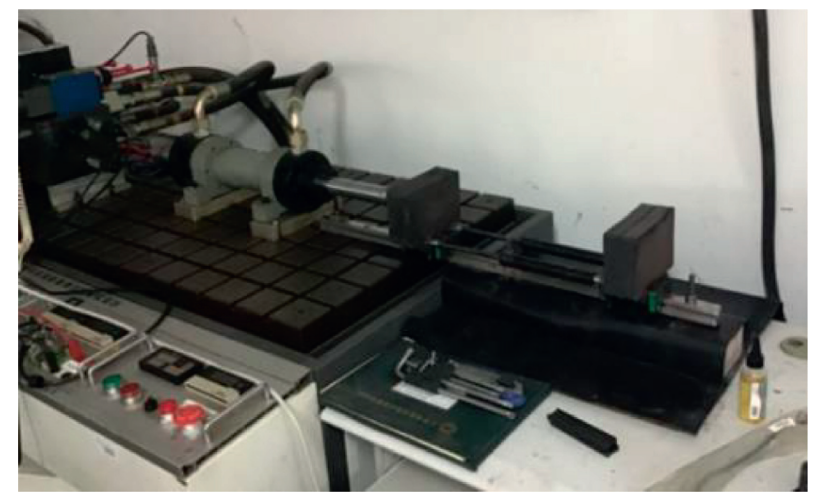

Figure 7: Two-mass connection hydraulic servo system.

TABLE 2: Hydraulic servo system parameters.

\begin{tabular}{lccc}
\hline Parameter name & Parameter symbol & Unit & Parameter value \\
\hline Area of rodless cavity & $A_{2}$ & $\mathrm{~m}^{2}$ & $1.256 \times 10^{-3}$ \\
Area of rod cavity & $A_{2}$ & $\mathrm{~m}^{2}$ & $3.14 \times 10^{-4}$ \\
Flow gain of hydraulic valve & $K_{q}$ & $\mathrm{~m}^{2} / \mathrm{s}$ & $\mathrm{MPa}$ \\
Effective bulk modulus of elasticity & $\beta_{e}$ & $\mathrm{~N} / \mathrm{m}$ & 690 \\
Stiffness of hydraulic spring & $K_{h}$ & $\mathrm{~N} / \mathrm{m}$ & $7.91 \times 10^{6}$ \\
& $K_{h}^{\prime}$ & $\mathrm{m}^{5} /(\mathrm{N} \cdot \mathrm{s})$ & $4.45 \times 10^{6}$ \\
Total flow pressure & $K_{c e}$ & $\mathrm{~m}^{5} /(\mathrm{N} \cdot \mathrm{s})$ & $10.9 \times 10^{-13}$ \\
Stiffness of flexible spring & $K_{c e}^{\prime}$ & $\mathrm{N} / \mathrm{m}$ & $8.11 \times 10^{-13}$ \\
Viscous damping coefficient & $K_{L}$ & $\mathrm{~N} /(\mathrm{m} / \mathrm{s})$ & $5 \times 10^{5}$ \\
Mass & $B_{L}$ & $\mathrm{~kg}$ & 100 \\
& $m_{L}$ & $\mathrm{~kg}$ & 8 \\
\hline
\end{tabular}

step convergence condition is relatively strict, and range of weights is usually limited based on the prior knowledge.

\section{Case Analysis}

CQYZ-MDH high-pressure and large-flow electrohydraulic servo performance experiment table is a new type of electrohydraulic valve experiment table designed according to the characteristics of ten-way hydraulic valve. In order to simulate the actual industrial pressure and flow, the maximum pressure can reach $21 \mathrm{MPa}$ and the maximum flow can reach $60 \mathrm{~L} / \mathrm{min}$. It is used to design the dynamic performance experiment of the servo system with numerical pressure less than $21 \mathrm{MPa}$. Based on this, the static and dynamic performance experiments of servo valve can also be completed. The experimental device is shown in Figure 7, and its specific parameters are shown in Table 2. The flexible components used for the connection of the hydraulic servo system are two slender hydraulic damping rods, which can provide the load stiffness slightly less than hydraulic spring stiffness. The two ends of the flexible rod are, respectively, connected with the mass blocks at both ends.

The principle of the test system is shown in Figure 8, which is composed of hydraulic valve, computer, PCI8335A$\mathrm{A} / \mathrm{D}, \mathrm{D} / \mathrm{A}$ acquisition card, proportional amplifier, flowmeter, sensor, and regulated power supply $(+24 \mathrm{~V})$. It can implement the function of data acquisition and processing and draw the processed image. To automate the testing process, the

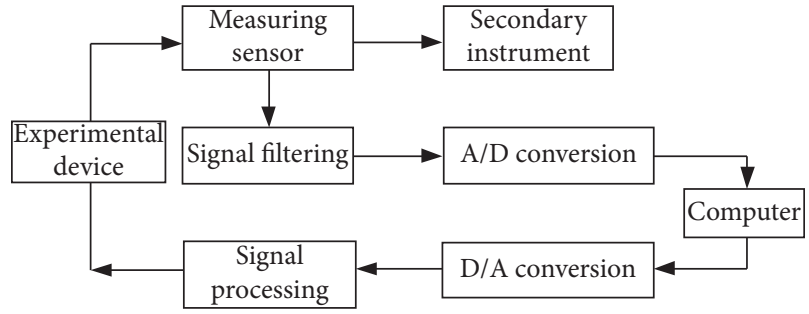

FIgURE 8: The principle of the test system.

computer outputs control signals and controls the hydraulic system. As shown in Figure 9, the multifunction data acquisition card of PCI8335A has the functions such as digital input, analog input and output, and counter.

Simulation Analysis. When the step $\mu$ is 0.002 , the expected response of the input adaptive controller is $2 \sin 12 t\left(\mathrm{~m} / \mathrm{s}^{2}\right)$. Figure 10 is the comparison diagram of input signal, fuzzy PID control, and adaptive control output signal, and Figure 11 is the error convergence comparison curve. It is seen that the output curve of adaptive controller is basically consistent with the output after reaching stability, while the fuzzy PID control has a certain amplitude attenuation and phase lag, which needs a longer stability time and exists a certain amount of error.

When the step $\mu$ is increased to 0.005 , the input signal response is still $2 \sin 12 t\left(\mathrm{~m} / \mathrm{s}^{2}\right)$. Figure 12 is the diagram of 


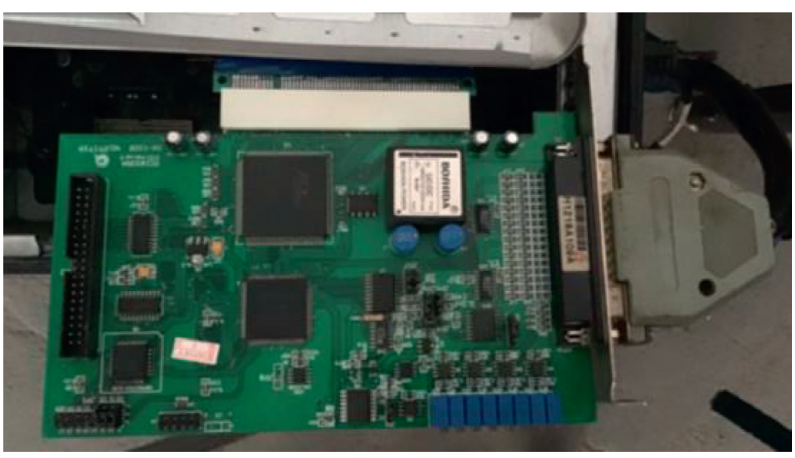

Figure 9: PCI8335A acquisition card.

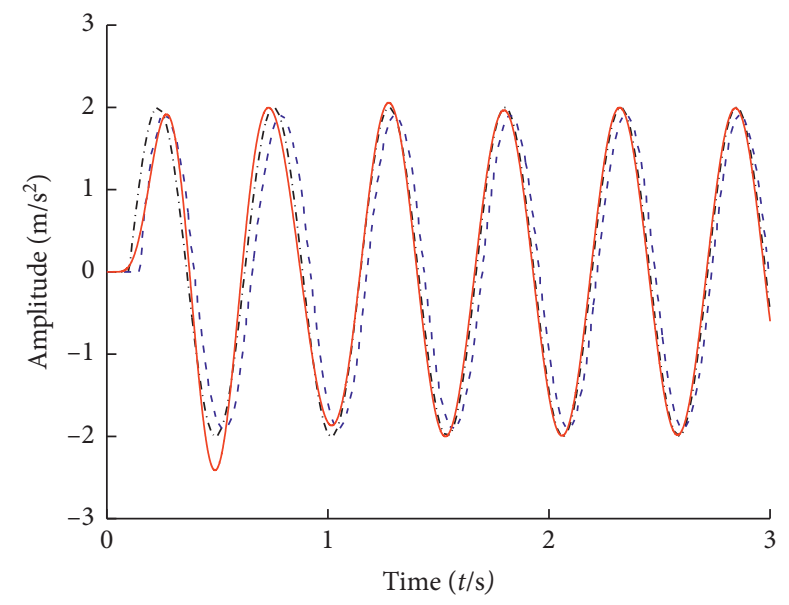

- . Input signal

- - - Fuzzy PID control output

- Adaptive control output

Figure 10: Sine signal before and after control.

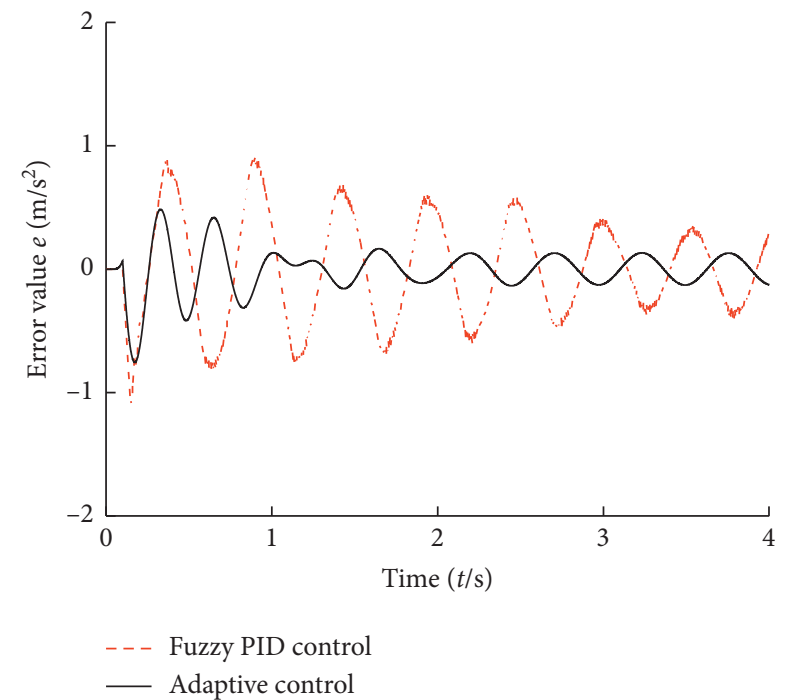

FIGURE 11: Error curve comparison. 


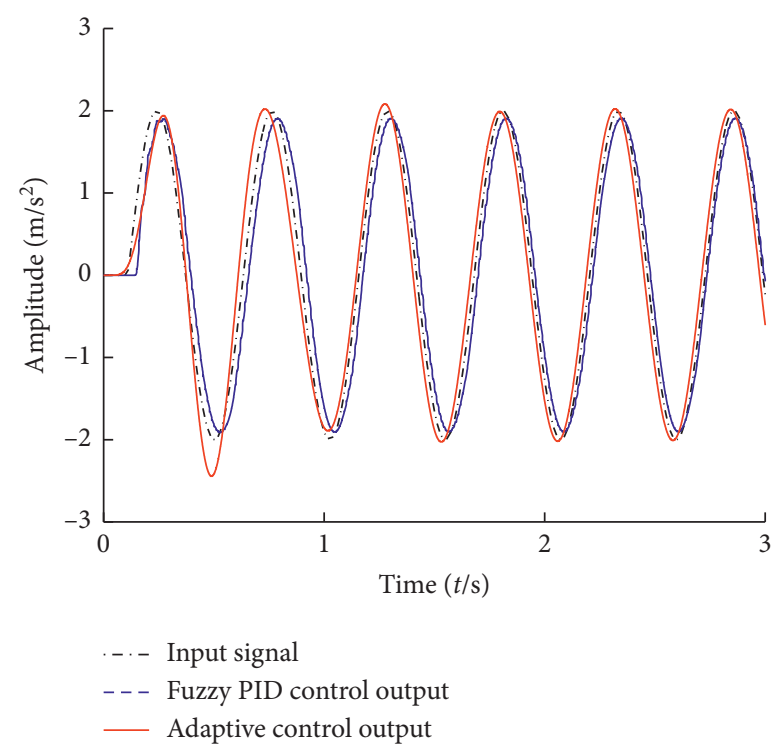

Figure 12: Signal comparison before and after control.

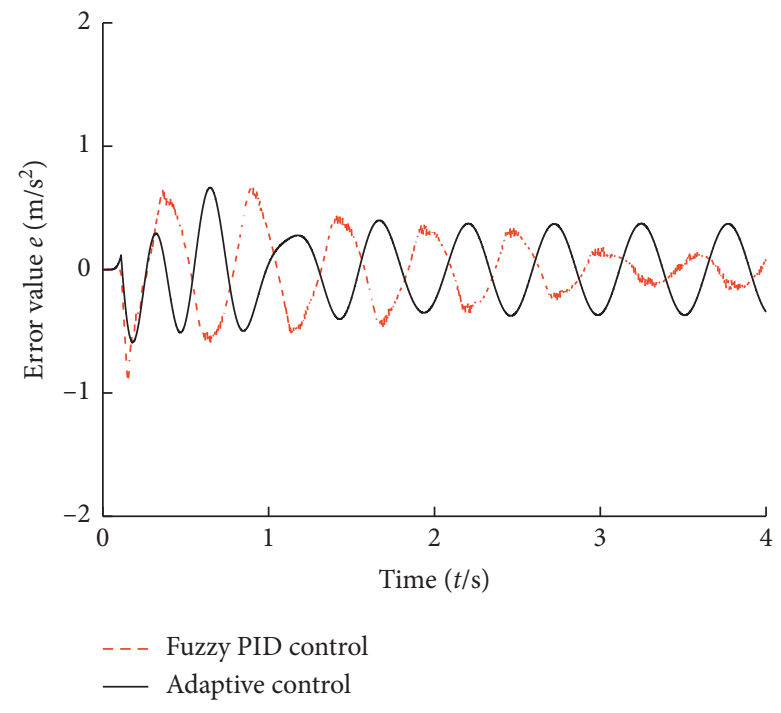

Figure 13: Error curve comparison.

curve after control, and the error convergence comparison curve is shown in Figure 13. The output curve of fuzzy PID control has little change, but the adaptive control output has a certain distortion, and the difference becomes larger, which indicates that the mean square error has increased significantly at this time. It is concluded that a larger step size should be chosen carefully; otherwise, a larger gradient noise will be produced.
Experimental Analysis. When the input signal is $2 \sin t(\mathrm{~V})$ test signal, the step parameter is 0.0003 , and Figure 14 is the comparison diagram before and after control. It is seen from the figure that the adaptive controller has good control performance, quickly reaches the steady state, and better reflects the input signal than the fuzzy PID algorithm. The fuzzy PID controller has a certain error value at the peak value. From Figure 15, with the continuous adjustment of 


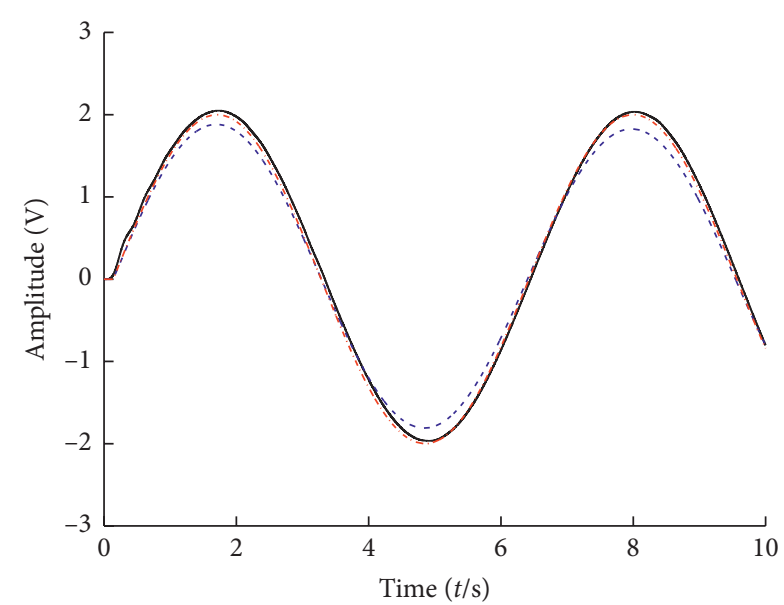

- Adaptive control output

- - - Fuzzy PID control output

-..- Input signal

FIGURE 14: Sine signal before and after control.

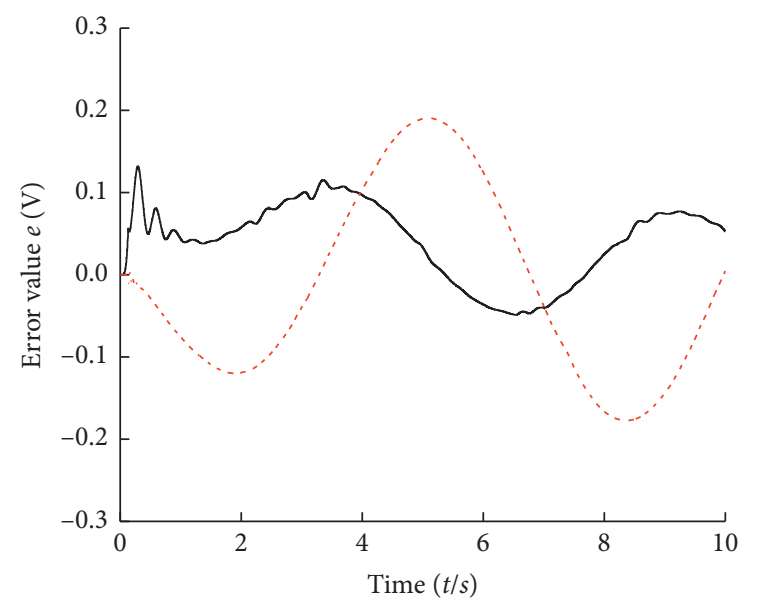

- Adaptive control

- - - Fuzzy PID control

Figure 15: Error curve comparison.

adaptive control, the instantaneous value of error decreases rapidly which reaches a stable state and does not fluctuate in a small range. However, the error of fuzzy PID control is relatively large.

\section{Conclusions}

The model of hydraulic servo actuators and flexible connection systems is the most commonly used model in this field. It is usually regarded as a rigid connection for the convenience of modeling and calculation. However, there are many complex modeling states in the actual system that cannot be ignored. The system is usually affected by nonlinear factors and coupling, which lead to distortion and a large error in the input signal.

In this paper, an adaptive controller is used to control and adjust the output signal response. The formula shows that the parameter selection of the step size has a great influence on the control accuracy. The accuracy of the output signal is improved by choosing the step size appropriately. Based on simulation and experiment, the adaptive algorithm exhibits better control precision than the fuzzy PID algorithm.

However, there are still some shortcomings in this paper; the hydraulic servo system is only a single-degree-of-freedom platform. For complex hydraulic servo mechanisms, such as electrohydraulic servo shaking tables with multiple degrees of freedom, due to the existence of multiple hydraulic cylinders and redundancy, the output signal is not only distorted but may also exhibit multiple harmonics. Therefore, the algorithm needs to be verified by a greater number of experimental platforms.

\section{Abbreviations}

$A_{1}$ : $\quad$ Effective area of rodless cavity, $\left(\mathrm{m}^{2}\right)$

$A_{2}$ : $\quad$ Effective area of rod cavity $\left(\mathrm{m}^{2}\right)$

$B_{L}$ : Viscous damping coefficient $(\mathrm{N} /(\mathrm{m} / \mathrm{s}))$

$C_{e p}: \quad$ Leakage coefficient $\left(\mathrm{m}^{3} / \mathrm{s} / \mathrm{Pa}\right)$

$C_{i p}: \quad$ Internal leakage coefficient $\left(\mathrm{m}^{3} / \mathrm{s} / \mathrm{Pa}\right)$

$C_{t c}: \quad$ Total leakage coefficient $\left(\mathrm{m}^{3} / \mathrm{s} / \mathrm{Pa}\right)$

$J(n)$ : Mean square error

$J(\infty)$ : Final solution

$K_{c}: \quad$ Flow-pressure coefficient, $K_{c}=\left(\partial q_{L} / \partial p_{L}\right),\left(\mathrm{m}^{3} / \mathrm{s} /\right.$

$K_{c e}: \quad$ Total flow-pressure coefficient, $K_{c e}=K_{c}+C_{t e},\left(\mathrm{~m}^{3} /\right.$ $\mathrm{s} / \mathrm{Pa})$

$K_{h}: \quad$ Hydraulic spring stiffness, $K_{h}=\left(4 \beta_{e} A_{1}^{2} / V_{e}\right),(\mathrm{N} / \mathrm{m})$

$K_{L}: \quad$ Connection stiffness $(\mathrm{N} / \mathrm{m})$

$K_{q}: \quad$ Flow gain, $K_{q}=\left(\partial q_{L} / \partial x_{v}\right),\left(\mathrm{m}^{2} / \mathrm{s}\right)$

$m_{L} \quad$ Mass $(\mathrm{kg})$

$m_{p}:$

$n: \quad$ The number of iteration

p: $\quad$ Cross correlation vector between tap input and expected response

$p_{1}$ : $\quad$ Pressure of rodless cavity $(\mathrm{Pa})$

$p_{2}$ : $\quad$ Pressure of rod cavity $(\mathrm{Pa})$

$p_{L}: \quad$ Load pressure drop $(\mathrm{Pa})$

$q_{1}$ : $\quad$ Flow rate into rodless cavity $(\mathrm{L} / \mathrm{min})$

$q_{2}$ : Outflow flow rate of rod cavity $(\mathrm{L} / \mathrm{min})$

$q_{L}$ : Load flow $\left(\mathrm{m}^{3} / \mathrm{s}\right)$

R: $\quad$ Correlation matrix of tap vector

$V_{1}$ : Liquid volume of rodless chamber $\left(\mathrm{m}^{3}\right)$

$V_{2}$ : Liquid volume of rod cavity $\left(\mathrm{m}^{3}\right)$

$V_{e}: \quad$ Equivalent volume $\left(\mathrm{m}^{3}\right)$

$x_{\mathrm{v}}: \quad$ Spool displacement $(\mathrm{m})$

$y_{L}: \quad$ Load displacement $(\mathrm{m})$

$y_{p}: \quad$ Piston displacement $(\mathrm{m})$

$\beta_{e}: \quad$ Equivalent bulk modulus of elasticity $(\mathrm{Pa})$

$\mu$ : $\quad$ Step parameter, a normal number

$\varepsilon_{0}(n)$ : The weight error vector.

\section{Data Availability}

The figures and equipment parameter data used to support the findings of this study are included within the article. 


\section{Conflicts of Interest}

The authors declare that they have no potential conflicts of interest with respect to the research, authorship, and/or publication of this article.

\section{Acknowledgments}

This project was supported by the HTC $3250 \mu \mathrm{n}$ precision NC lathe, and turning center is a subproject attached to the National Science and Technology Major Project (2009ZX04001-053).

\section{References}

[1] M.-H. Chiang, "A novel pitch control system for a wind turbine driven by a variable-speed pump-controlled hydraulic servo system," Mechatronics, vol. 21, no. 4, pp. 753-761, 2011.

[2] M.-H. Chiang and B.-Y. Wu, "Intelligent position control of a variable rotational speed hydraulic pump-controlled system," Advanced Science Letters, vol. 13, no. 1, pp. 436-441, 2012.

[3] K. Szabat and T. Orlowska-Kowalska, "Performance improvement of industrial drives with mechanical elasticity using nonlinear adaptive Kalman filter," IEEE Transactions on Industrial Electronics, vol. 55, no. 3, pp. 1075-1084, 2008.

[4] H. Du, Y. H. Chen, S. W. Huang, M. H. Wu, H. Huang, and Y. Z. Li, "Pressure control of electro-hydraulic servo loading system in heavy vehicle steering testboard based on integral sliding mode control," Proceedings of the Institution of Mechanical Engineers, Part D: Journal of Automobile Engineering, vol. 243, no. 2-3, pp. 458-468, 2019.

[5] M. Miroslav, N. Vlastimir, and A. Dragan, "Position control of an electro-hydraulic servo system using sliding mode control enhanced by fuzzy PI controller," Mechanical Engineering, vol. 9, pp. 1217-1230, 2002.

[6] A. R. Plummer, "High bandwidth motion control for multiaxis servohydraulic mechanisms," in Proceedings of the 2007 ASME International Mechanical Engineering Congress and Exposition, pp. 1-7, American Society of Mechanical Engineers, Seattle, WA, USA, November 2007.

[7] N. E. Earnhart and K. A. Cunefare, "Compact Helmholtz resonators for hydraulic systems," International Journal of Fluid Power, vol. 13, no. 1, pp. 41-50, 2012.

[8] D.-H. Lee, J. H. Lee, and J.-W. Ahn, "Mechanical vibration reduction control of two-mass permanent magnet synchronous motor using adaptive notch filter with fast Fourier transform analysis," IET Electric Power Applications, vol. 6, no. 7, pp. 455-461, 2012.

[9] W. Y. Wang and A. W. Shen, "Detection and reduction of middle-frequency resonance for industrial servo with selftuning lowpass filter," Journal of Control Science and Engineering, vol. 21, no. 7, pp. 899-907, 2013.

[10] Y. Tang, Z. Zhu, and G. Shen, "Design and experimental evaluation of feedforward controller integrating filtered-x LMS algorithm with applications to electro-hydraulic force control systems," Proceedings of the Institution of Mechanical Engineers, Part C: Journal of Mechanical Engineering Science, vol. 230, no. 12, pp. 1951-1967, 2016.

[11] R. K. Agarwal, I. Hussain, and B. Singh, "Application of LMSbased NN structure for power quality enhancement in a distribution network under abnormal conditions," IEEE Transactions on Neural Networks and Learning Systems, vol. 29, no. 5, pp. 1598-1607, 2018.
[12] K. B. Lee, J. H. Song, I. Choy, and J. Y. Yoo, "An inertia identification using ROELO for low speed control of electric machine," in Proceedings of the IEEE Applied Power Electronics Conference \& Exposition, pp. 1052-1055, IEEE, Miami Beach, FL, USA, February 2003.

[13] T. Umeno and Y. Hori, "Robust speed control of DC servomotors using modern two-degrees-of-freedom controller design," IEEE Transactions on Industrial Electronics, vol. 38, pp. 363-368, 2002.

[14] M. Nayyerloo, J. G. Chase, G. A. Macrae, X. Chen, and C. E. Hann, "Structural health monitoring using adaptive LMS filters," International Journal of Computer Applications in Technology, vol. 39, no. 1-3, pp. 130-136, 2010.

[15] C. Guan and S. A. Zhu, "Multiple sliding mode robust adaptive control of an electro-hydraulic servo system," Control Theory and Application, vol. 22, no. 6, pp. 931-938, 2005.

[16] R. Ghazali, Y. M. Sam, M. F. Rahmat, A. W. I. M. Hashim, and Zulfatman, "Position tracking control of an electro-hydraulic servo system using sliding mode control," in Proceedings of the 2010 IEEE Student Conference on Research and Development, Institute of Electrical and Electronics Engineers Inc, Kuala Lumpur, Malaysia, December 2010.

[17] B. Yu, R. Liu, Q. Zhu, Z. Huang, Z. Jin, and X. Wang, "Highaccuracy force control with nonlinear feedforward compensation for a hydraulic drive unit," IEEE Access, vol. 7, no. 99, pp. 101063-101072, 2019.

[18] J. Carvajal, G. Chen, and H. Gmen, "Fuzzy PID controller: design, performance evaluation, and stability analysis," Information Sciences, vol. 123, no. 3-4, pp. 249-270, 2000.

[19] V. K. P. Mittal, "Parallel fuzzy P+fuzzy I+fuzzy D controller: design and performance evaluation," International Journal of Automation«Computing, vol. 7, no. 4, pp. 463-471, 2010.

[20] I. Pan, S. Das, and A. Gupta, "Tuning of an optimal fuzzy PID controller with stochastic algorithms for networked control systems with random time delay," ISA Transactions, vol. 50, no. 1, pp. 28-36, 2010.

[21] X. K. Wang, Z. L. Sun, L. Wang, and D. Q. Feng, "Design and Research Based on Fuzzy PID-Parameters Self-Tuning Controller with MATLAB," in Proceedings of the 2008 International Conference on Advanced Computer Theory and Engineering, IEEE Computer Society, Phuket, Thailand, December 2008.

[22] M. E. A. Mohamed and Y. Guo, "Separately excited DC motor speed tracking control using adaptive neuro-fuzzy inference system based on genetic algorithm particle swarm optimization and fuzzy auto-tuning PID," in Proceedings of the $3 \mathrm{rd}$ International Symposium on Resource Exploration and Environmental Science, pp. 27-28, IOP Publishing Ltd, Ordos, China, April 2019.

[23] Y. Minagawa, E. Kikutani, and S. Kurokawa, "Study of transverse bunch-by-bunch feedback systems based on the two tap FIR filter," Nuclear Instruments \& Methods in Physics Research, Section A, (Accelerators, Spectrometers, Detectors and Associated Equipment), vol. 416, no. 2-3, pp. 193-209, 1998.

[24] M. Tobiyama, E. Kikutani, and T. Obina, "Initial test of a bunch feedback system with a two-tap FIR filter board," American Institute of Physics, vol. 390, pp. 359-367, 1997.

[25] D. Zhang, Z. Wang, and M. Tomizuka, "A variable-parameter-model-based feedforward compensation method for tracking control," IEEE/CAA Journal of Automatica Sinica, vol. 7, no. 3, pp. 693-701, 2020. 
[26] J. Wang and G. Liu, "A novel firefly algorithm with selfadaptive step strategy," International Journal of Innovative Computing and Applications, vol. 10, no. 1, pp. 18-30, 2019.

[27] S. Haykin, Adaptive Filter Theory, Prentice-Hall, Inc., Upper Saddle River, NJ, USA, 5th edition, 2014.

[28] D. Bismor, "Extension of LMS stability condition over a wide set of signals," International Journal of Adaptive Control and Signal Processing, vol. 29, no. 5, pp. 653-670, 2015.

[29] M. Moinuddin and A. Zerguine, "Steady-state analysis of the Normalized Least Mean Fourth algorithm without the independence and small step size assumptions," in Proceedings of the IEEE International Conference on Acoustics, pp. 3097-3100, Institute of Electrical and Electronics Engineers Inc, Taipei, China, April 2009. 